Presence of ET was ascertained through telephone interview, physical examination or study of medical records. Additionally, medical records from a records-linkage system were examined for the participants from Olmsted County, MN. In the population-based sample, relatives of patients with PD were slightly more likely to have tremor than were relatives of controls (hazard ratio $1.51 ; P=0.08$ ); however, the risk was significantly increased for relatives of patients with $P D$ onset in the lowest age tertile ( $\leq 66$ years; hazard ratio 2.24; $P=0.006$ ). Decreasing age of onset of PD also correlated with increasing risk of ET among relatives in the referral-based sample (linear trend over three age tertiles; $P=0.001$ ). Being a man or having a relative with a tremorpredominant or mixed form of PD (as opposed to the akinetic-rigid form) also conferred an increased risk of ET. Furthermore, relatives of referred patients had a greater risk of ET than did relatives of population-based patients.

Original article Rocca WA et al. (2007) Increased risk of essential tremor in first-degree relatives of patients with Parkinson's disease. Mov Disord [doi: 10.1002/mds.21584]

\section{Folic acid supplementation: effective for primary prevention of stroke}

Folic acid is an important regulator of homocysteine metabolism, and evidence suggests that folate supplementation might reduce the risk of stroke; however, studies examining the effects of folate supplementation on other cardiovascular end points have produced conflicting results. A recent meta-analysis by Wang and co-workers has assessed whether folic acid supplementation can prevent stroke.

The authors identified eight randomized clinical trials that compared folic acid supplementation with one of three alternative regimens: placebo, usual care, or supplementation with a lower dose of folic acid. Three trials were conducted in the USA or Canada, where folic acid fortification of grain products was fully implemented by 1998 . Four trials were conducted in regions with unfortified grain, and one was conducted in a partially fortified population. Overall, folic acid supplementation was associated with a significant reduction in the risk of stroke (relative risk [RR] 0.82, 95\% $\mathrm{Cl}$ 0.68-1.00; $P=0.045)$. The relationship seemed to be causal, because longer duration of supplementation was associated with greater reduction in the risk of stroke (RR 1.00 for interventions of $\leq 36$ months vs 0.71 for interventions $>36$ months). Folic acid supplementation had a smaller beneficial effect on the risk of stroke in regions with folic acid fortification of grain than in regions with no or partial fortification. Supplementation did not reduce risk of stroke in patients with previous history of stroke, but was effective for primary prevention (RR $0.75,95 \% \mathrm{Cl} 0.62-0.94$; $P=0.002$ ).

Original article Wang X et al. (2007) Efficacy of folic acid supplementation in stroke prevention: a meta-analysis. Lancet 369: 1876-1882

\section{Peripheral-leukocyte profiles could be pharmacologic biomarkers for AD}

Circulating leukocytes are potential biomarkers of CNS disease and could provide insight into pharmacologic activity in neurodegenerative diseases. In a recently published study, Mhyre et al. identified a number of leukocyte proteins that are differentially expressed in peripheral leukocytes of patients with Alzheimer's disease (AD) before and after therapy with valproic acid (VPA).

Protein expression profiles of peripheral leukocytes were classified in 15 patients with moderate AD treated with VPA, which has been shown to be neuroprotective in $A D$ although its mechanism of action remains unclear. Gel electrophoresis analysis of peripheralblood samples collected at baseline and after 3-4 weeks of treatment revealed changes in the level of expression of 10 proteins in treated subjects; two of these proteins were upregulated and eight were downregulated in response to VPA. Nine potential biomarkers were identified, which belonged to various functional classes. Alterations in transcript and protein expression were validated in a subset of the potential biomarkers in both peripheral-blood samples and cultures of human peripheral blood mononuclear cells. The precise changes to these markers induced by the therapeutic action of VPA therapy are not known, but the authors propose that the similarities between the in vivo and in vitro data suggest that these markers are generalizable VPA targets.

The authors conclude that differential protein expression profiles in peripheral leukocytes 